\title{
Dexamethasone in adults with bacterial meningitis
}

De Gans J, van de Beek D, for the European Dexamethasone in Adulthood Bacterial Meningitis Study Investigators. Dexamethasone in adults with bacterial meningitis. $N$ Engl $\mathcal{F}$ Med 2002;347(20):1549-56.

Background: Even when bacterial meningitis is diagnosed and treated promptly, it causes significant morbidity and mortality in adults. Dexamethasone, which lessens cerebrospinal inflammation, has been shown to reduce the incidence of hearing loss in children with meningitis caused by Haemophilus influenzae type b and possibly Streptococcus pneumoniae. ${ }^{1}$ To date, strong evidence to support the use of steroids in adults with bacterial meningitis has been lacking.

Question: Is the adjunctive use of dexamethasone beneficial in the treatment of adults with acute bacterial meningitis?

Design: This prospective, randomized, double-blind, placebo-controlled multicentre trial, conducted in Europe between June 1993 and December 2001, enrolled 301 patients aged 17 years or more with suspected bacterial meningitis (based on the results of cerebrospinal fluid $[\mathrm{CSF}]$ analysis). Patients were randomly assigned to receive either dexamethasone, $10 \mathrm{mg}$ intravenously, or a placebo before or with the first dose of intravenous antibiotics and every 6 hours thereafter for a total of 4 days.

Among the exclusion criteria were hypersensitivity to study medications, pregnancy, presence of a cerebrospinal shunt and receipt of systemic antibiotics within the previous 48 hours. The primary outcome measure was the subjects' score on the Glasgow Outcome Scale (GOS) 8 weeks after enrolment. The GOS is a validated scoring system for neurologic disability, with scores ranging from 1 (death) to 5 (mild or no disability). Secondary outcomes included death, focal neurologic abnormalities, hearing loss and clinically significant gastrointestinal bleeding. Outcomes were analyzed on an intention-to-treat basis, with the last observation carried forward for subjects who died or were lost to follow-up. Subgroup analysis by infecting organism was also performed.

Results: Baseline demographic, clinical and CSF characteristics of the dexamethasone $(n=157)$ and placebo $(n=$ 144) groups were comparable, with the exception that a higher percentage of patients in the dexamethasone group had seizures on presentation $(10 \%$ v. $5 \%$ in the placebo group). All 301 subjects enrolled received their assigned initial treatments and were included in the final analysis. Of 269 survivors, including 11 in each group who were withdrawn early for various reasons, 262 were assessed at 8 weeks. Only 7 subjects were lost to follow-up. Initial antimicrobial therapy was at least $97 \%$ effective in the 2 groups.

At 8 weeks, $15 \%$ of the patients in the dexamethasone group and $25 \%$ in the placebo group had an unfavourable neurological outcome, defined as a GOS score of 1 to 4 (relative risk [RR] 0.59, 95\% confidence interval [CI] 0.37-0.94; $p=0.03$ ). Coma, hypotension and pneumococcal meningitis were significant predictors of an unfavourable outcome. Mortality was lower in the dexamethasone group ( $7 \%$ v. $15 \%$ : RR $0.48,95 \%$ CI $0.24-0.96 ; p=0.04$ ), as was the incidence of impaired consciousness, seizures and cardiorespiratory failure while in hospital. In the subgroup analysis, a significant reduction in unfavourable outcomes was seen only in patients with pneumococcal meningitis, particularly those with moderate-to-severe disease. The incidence of adverse events, including significant gastrointestinal bleeding, was similar in both groups. Steroids did not reduce the incidence of neurologic sequelae, including hearing loss.

Commentary: This is the first prospective clinical trial that strongly supports the use of dexamethasone in adults with acute bacterial meningitis, albeit in select circumstances. The benefits were limited to patients with meningitis caused by $S$. pneumoniae. No apparent benefit was noted in the small number of patients with meningococcal meningitis. Because the use of high-dose steroids in sepsis has been associated with increased mortality, adjunctive steroid therapy cannot be recommended in patients with meningitis who also show signs of severe sepsis.

A number of important questions remain unanswered. Delayed clinical improvement in the placebo group beyond the 8-week follow-up period would decrease the observed treatment effect. The optimal duration of steroid therapy in acute bacterial meningitis is unknown, and it is conceivable that shorter courses may be as effective as a 4-day course. Finally, because steroids may impair the penetration of vancomycin into the CSF, their use in meningitis caused by penicillin-resistant $S$. pneumoniae could potentially be detrimental and warrants further study.

Practice implications: Dexamethasone appears to be a safe and beneficial adjunctive therapy in the treatment of moderate-to-severe acute bacterial meningitis caused by $S$. pneumoniae. A 10-mg dose should be administered intravenously just before or with the first dose of antibiotics in adults who present with suspected bacterial meningitis without systemic sepsis and should be continued for 4 days in those with pneumococcal disease. Dexamethasone should be discontinued if another microbiological cause of meningitis is confirmed.

\section{Kathryn N. Suh \\ Division of Infectious Diseases \\ Queen's University \\ Kingston, Ont.}

In the Literature is edited by Dr. Donald Farquhar, head of the Division of Internal Medicine, Queen's University, Kingston, Ont.

\section{References}

1. McIntyre PB, Berkey CS, King SM, Schaad UB, Kilpi T, Kanra GY, et al. Dexamethasone as adjunctive therapy in bacterial meningitis. A metaanalysis of randomized clinical trials since 1988. 7AMA 1997;278(11):925-31.

2. Cronin L, Cook DJ, Carlet J, Heyland DK, King D, Lansang MA, et al. Corticosteroid treatment for sepsis: a critical appraisal and meta-analysis of the literature. Crit Care Med 1995;23(8):1430-9. 